\title{
Contrary melanoma-associated antigen-A expression at the tumor front and center: A comparative analysis of stage I and IV head and neck squamous cell carcinoma
}

\author{
STEFAN HARTMANN ${ }^{1}$, MUNA BRISAM ${ }^{1}$, STEPHAN RAUTHE $^{2}$, OLIVER DRIEMEL ${ }^{3}$, ROMAN C. BRANDS $^{1}$, \\ ANDREAS ROSENWALD ${ }^{2}$, ALEXANDER C. KÜBLER ${ }^{1}$ and URS D. A. MÜLLER-RICHTER ${ }^{1}$ \\ ${ }^{1}$ Department of Oral and Maxillofacial Plastic Surgery, University Hospital Würzburg, D-97070 Würzburg; \\ ${ }^{2}$ Institute of Pathology and Comprehensive Cancer Center Mainfranken, University of Würzburg, D-97080 Würzburg; \\ ${ }^{3}$ Department of Oral and Maxillofacial Plastic Surgery, University Hospital Rostock, D-18057 Rostock, Germany
}

Received June 13, 2015; Accepted April 22, 2016

DOI: $10.3892 / \mathrm{ol} .2016 .4945$

\begin{abstract}
There is a growing body of evidence indicating that several melanoma-associated antigen-A (MAGE-A) subgroups contribute to the malignancy of head and neck cancer. The present study retrospectively analyzed the expression of all known MAGE-A subgroups in the tumor front and center of 38 head and neck cancer patients (Union for International Cancer Control stage I or IV) by immunohistochemistry. MAGE-A1, -A6, -A8, -A9 and -A11 were expressed at significantly higher levels at the tumor front of stage IV specimens compared with the tumor front of stage I specimens. In stage I cancer, the tumor center and front ratio $(\mathrm{C} / \mathrm{F}$ ratio) for each subgroup was $>1.0$. In stage IV cancer, the $\mathrm{C} / \mathrm{F}$ ratio was $<1.0$ in $9 / 11$ subgroups. The most significant change in the expression pattern was observed for MAGE-A11. These results indicated that there is a marked alteration and shift to the invasive front of almost all MAGE-A subgroups, but particularly MAGE-A11, during the progression of head and neck squamous cell carcinoma.
\end{abstract}

\section{Introduction}

The treatment of the advanced stages of head and neck squamous cell carcinoma remains challenging $(1,2)$. In particular, a lack of locoregional control and failure of neck dissection (occurrence or recurrence of lymph node metastases following neck dissection) often leads to fatal outcomes and a poor prognosis for the affected patients (3). Thus, the initial recognition

Correspondence to: Dr Stefan Hartmann, Department of Oral and Maxillofacial Plastic Surgery, University Hospital Würzburg, Pleicherwall 2, D-97070 Würzburg, Germany

E-mail: hartmann_s2@ukw.de

Key words: head and neck cancer, melanoma-associated antigen, tumor center, tumor front, A11 of patients with a higher risk of having these factors could improve the long-term outcomes by allowing a more aggressive treatment approach and a closer follow-up for that patient. Melanoma-associated antigen A (MAGE-A) was identified in the early 1990s by van der Bruggen et al (4). There is growing evidence that MAGE-A proteins are associated with or contribute to several solid malignancies, including head and neck cancer (5-7). This can be explained, at least in part, by the MAGE-A-mediated decrease in the cellular p53 levels, leading to chemoresistance (8). In this context, our previous studies investigated the contributions of MAGE-A expression to the reduced cytotoxicity of conventional therapies in head and neck cancer. Based on those studies, evidence was provided showing that several MAGE-A subgroups, particularly MAGE-A11, are associated with the decreased efficacy of cisplatin, 5-fluorouracil, docetaxel, paclitaxel, cetuximab, panitumumab, erlotinib and gefitinib $(9,10)$. However, these findings are limited to artificial cell culture systems, and clinical evidence is required to strengthen our hypotheses. Notably, it has also demonstrated that MAGE-A11 expression is correlated with a poorer prognosis for breast cancer (11). Similar results were reported by Minges et al for castration-recurrent prostate cancers (12).

In addition to the association between several subgroups and a poor prognosis, MAGE-A expression is also an important predictor of malignant transformation. A study by Ries et al clearly showed that MAGE-A expression is restricted to oral leukoplakia tissues that transform into invasive cancer, and is not present in leukoplakia without progression to malignancy (13). Complementing these findings, we previously showed that MAGE-A expression is found in leukoplakia with dysplasia and carcinoma in situ, but not in benign lesions, such as ulcers or epulis (14).

The present study was designed to quantify the expression of all known MAGE-A antigens at the tumor center and the tumor front with respect to the UICC stages of the patients investigated. The differences between the different sites (invasive front vs. tumor center), and between limited and advanced disease [Union for International Cancer Control (UICC) stage I vs. IV] were analyzed. In addition, 
the probable role of a cumulative MAGE-A expression score was assessed.

\section{Patients and methods}

Patients and tissues. The data investigated in this study were collected from patients with oral squamous cell carcinoma (OSCC) who were treated between August 2001 and August 2003 at the Department of Oral and Maxillofacial Plastic Surgery of the University Hospital Würzburg (Würzburg, Germany). The tissue samples were provided by the Institute of Pathology of the University of Würzburg (Würzburg, Germany). The tumor locations consisted of the lip, tongue, cheek, tonsil, palate and oropharynx. None of the tumors originated from the respiratory epithelium. After an initial screening, the patients were subdivided into two groups by clinically- or pathologically-confirmed tumor stage, which was determined according to the UICC criteria (15). The group with stage I cancers consisted of 23 patients (16 males and 7 females), with a mean age of 66.52 years [standard deviation (SD), \pm 13.95$]$. The group with stage IV cancers consisted of 15 patients (11 males and 4 females), with a mean age of 53.36 years $(\mathrm{SD}, \pm 9.95)$. The patients were classified as stage IV based on the presence of T4 (11 patients) or N2 (4 patients) disease. None of the patients had confirmed distant metastasis. The identification of the tumor center and invasive front was supported by an expert pathologist. The study was approved by the ethics committee of the University of Würzburg (reference, 2016050801 ).

Tissue microarrays (TMAs) and immunohistochemical staining. For TMA preparation, paraffin-embedded samples of the OSCCs were used. From each specimen, a sample of the tumor front and tumor center were constructed, represented by three $0.6-\mathrm{mm}$ cores with a thickness of $1 \mu \mathrm{m}$. The TMA were mounted on saline-coated slides. Phosphate-buffered saline was used as a diluent for the washing and rinsing steps throughout the protocol for TMA preparation. Following deparaffinization [with xylene and decreasing concentrations of ethanol (100-70\%)] and consecutive rehydration, antigen retrieval was performed by autoclaving the samples for $15 \mathrm{~min}$ in distinct saline buffers (Table I). Next, the sections were incubated with the MAGE-A primary antibodies (Table I) at room temperature for $60 \mathrm{~min}$. Subsequent to washing, the secondary antibody [ADVANCE ${ }^{\mathrm{TM}}$ Horseradish peroxidase (HRP) Link] was added for another $20 \mathrm{~min}$. ADVANCE HRP enzyme was then added for $20 \mathrm{~min}$. Afterwards, detection was performed with a Dako ADVANCE system and 3,3'-diaminobenzidine was used as the chromogen (all Pathology Products Dako Deutschland GmbH, Hamburg, Germany). Finally, the slides were counterstained with hematoxylin. Anonymized adult testis tissue from the Institute of Pathology of the University of Würzburg served as positive controls for immunohistochemical staining. Lung and testis tissues without secondary antibody staining served as negative controls.

Semiquantitative staining score. To express the amount of staining, a semiquantitative method (immunoreactive score) was used, as described previously (16). For each section (tumor front or center) a multiplicative score was determined. The immunoreactivity score (IRS) with arbitrary units was calculated by multiplying the amount of staining by the percentage of positive cells (Table II). Based on the scoring system provided in Table II, the score of each specimen ranged between 0 and 12 arbitrary units. In addition to rating the tumor center and the invasive front separately, the total expression for every subgroup and patient was also summed up (IRS total = IRS invasive front + IRS tumor center).

Statistical analysis. For the statistical analysis and visualization of the data, the GraphPad Prism 6.04 software program (GraphPad Software Inc., La Jolla, CA, USA) was utilized. Due to the number of biopsies, differences between the stage I and IV group specimens were analyzed by the two-tailed Mann-Whitney test. $\mathrm{P} \leq 0.05$ was used to indicate a statistically significant difference.

\section{Results}

MAGE-A expression is found in the invasive front and the tumor center. In general, different expression levels of all MAGE-A subgroups were detected in the stage I and IV cancers (Figs. 1 and 2). In the stage I group, the lowest expression in the tumor center was found for MAGE-A1 (2.26) and the highest expression was found for MAGE-A6 (7.35). At the invasive front, the lowest expression was again found for MAGE-A1 (1.22), while the highest expression was observed for MAGE-A3 (4.87). In contrast to these findings, in the stage IV group, MAGE-A5 showed the highest expression at the tumor center (6.8) and MAGE-A11 showed the lowest expression (1.53). Regarding the invasive front, MAGE-A11 showed the lowest expression (2.27) and MAGE-A6 showed the highest expression (7.4) (Tables III and IV). Every MAGE-A subgroup was found to be expressed in all four investigated groups (invasive front and center in stages I and IV). In the stage I group, the mean cumulative MAGE-A expression ranged from 3.48 for MAGE-A1 to 11.57 for MAGE-A6. By contrast, the lowest mean cumulative MAGE-A expression in the stage IV group was observed for MAGE-A11 (3.8). MAGE-A6 yielded the highest mean cumulative expression (13.0).

Cumulative MAGE-A expression score is not associated with different UICC stages. The cumulative MAGE-A expression score in the UICC stage I cancers was 85.87 (SD, \pm 51.76$)$ and that in stage IV cancers was 98.67 (SD, \pm 40.26$)$. The Mann-Whitney test used to compare the groups showed no significant differences in the expression scores between stage I and stage IV cancers $(\mathrm{P}=0.5014)$.

$M A G E-A$ expression at the tumor center shows a tendency to differ between UICC stages I and IV. To compare the expression of the different subgroups in the tumor center between stage I and stage IV cancers, the Mann-Whitney test was used. The MAGE-A1, -A3 and -A5 subgroups were more strongly expressed in UICC stage IV cancers compared with UICC stage I tumors. However, no subgroup showed significantly different expression at the tumor center in a comparison of stage I and stage IV cancers. For MAGE-A1, a trend could be observed, but this was not statistically significant $(\mathrm{P}=0.0537)$ (Table III). 
Table I. Origin, dilution and source of the MAGE-A antibodies used in the study.

\begin{tabular}{|c|c|c|c|c|}
\hline Antigen & Origin & Heat buffer & Dilution & Source \\
\hline MAGE-A1 & Rabbit & $\mathrm{CA}$ & $1: 100$ & Antibodies online GmbH (Aachen, Germany) \\
\hline MAGE-A2 & Rabbit & $\mathrm{TR}$ & $1: 20$ & Santa Cruz Biotechnology, Inc. (Dallas, TX, USA) \\
\hline MAGE-A3 & Rabbit & $\mathrm{CA}$ & $1: 100$ & Novus Biologicals (Littleton, CO, USA) \\
\hline MAGE-A4 & Rabbit & $\mathrm{TR}$ & $1: 40$ & Abnova (Taipei, Taiwan) \\
\hline MAGE-A5 & Rabbit & $\mathrm{CA}$ & $1: 100$ & Antibodies online GmbH (Aachen, Germany) \\
\hline MAGE-A6 & Rabbit & $\mathrm{CA}$ & $1: 100$ & Antibodies online GmbH (Aachen, Germany) \\
\hline MAGE-A8 & Rabbit & $\mathrm{TR}$ & $1: 30$ & Santa Cruz Biotechnology, Inc. (Dallas, TX, USA) \\
\hline MAGE-A9 & Rabbit & $\mathrm{TR}$ & $1: 10$ & Santa Cruz Biotechnology, Inc. (Dallas, TX, USA) \\
\hline MAGE-A10 & Rabbit & $\mathrm{TR}$ & $1: 10$ & Abnova (Taipei, Taiwan) \\
\hline MAGE-A11 & Rabbit & $\mathrm{TR}$ & $1: 40$ & Santa Cruz Biotechnology, Inc. (Dallas, TX, USA) \\
\hline MAGE-A12 & Rabbit & TR & $1: 10$ & Abnova (Taipei, Taiwan) \\
\hline
\end{tabular}

CA, citric acid (pH, 6.0); TR, Target Retrieval (pH, 6.1); MAGE, melanoma-associated antigen-A.

Table II. Characteristics of the semiquantitative immunohistochemical staining score.

\begin{tabular}{ll}
\hline Score & \multicolumn{1}{c}{ Characteristic } \\
\hline Amount of staining & \\
0 & No reaction \\
1 & Weak reaction \\
2 & Modest reaction \\
3 & Strong reaction \\
Percentage of positive cells & \\
0 & Negative \\
1 & $<10 \%$ positive cells \\
2 & $10-50 \%$ positive cells \\
3 & $51-80 \%$ positive cells \\
4 & $>80 \%$ positive cells \\
\hline
\end{tabular}

Table III. Comparison of the MAGE-A expression at the tumor center using the Mann-Whitney test.

\begin{tabular}{lccc}
\hline $\begin{array}{l}\text { MAGE-A } \\
\text { subgroup }\end{array}$ & UICC stage I & UICC stage IV & P-value \\
\hline A1 & 2.26 & 4.53 & 0.0537 \\
A2 & 5.48 & 4.87 & 0.6158 \\
A3 & 5.13 & 5.67 & 0.6272 \\
A4 & 4.35 & 3.27 & 0.8173 \\
A5 & 5.57 & 6.80 & 0.4563 \\
A6 & 7.35 & 5.60 & 0.1457 \\
A8 & 4.57 & 2.53 & 0.4922 \\
A9 & 4.04 & 2.13 & 0.4953 \\
A10 & 5.13 & 3.67 & 0.4953 \\
A11 & 3.39 & 1.53 & 0.1256 \\
A12 & 5.04 & 4.27 & 0.6494 \\
\hline
\end{tabular}

MAGE, melanoma-associated antigen-A; UICC, Union for International Cancer Control.
Table IV. Comparison of the MAGE-A expression at the invasive front using the Mann-Whitney test.

MAGE-A

subgroup UICC stage I UICC stage IV P-value

\begin{tabular}{llll}
\hline A1 & 1.22 & 3.00 & $0.0400^{\mathrm{a}}$ \\
$\mathrm{A} 2$ & 3.04 & 5.20 & 0.1263 \\
$\mathrm{~A} 3$ & 4.87 & 5.87 & 0.4067 \\
$\mathrm{~A} 4$ & 2.83 & 4.40 & 0.1032 \\
$\mathrm{~A} 5$ & 4.30 & 5.27 & 0.3014 \\
A6 & 4.22 & 7.40 & $0.0331^{\mathrm{a}}$ \\
$\mathrm{A} 8$ & 2.04 & 3.93 & $0.0434^{\mathrm{a}}$ \\
$\mathrm{A} 9$ & 1.87 & 5.07 & $0.0060^{\mathrm{a}}$ \\
$\mathrm{A} 10$ & 4.57 & 5.93 & 0.2370 \\
$\mathrm{~A} 11$ & 0.74 & 2.27 & $0.0249^{\mathrm{a}}$ \\
$\mathrm{A} 12$ & 3.87 & 5.47 & 0.2213
\end{tabular}

${ }^{\mathrm{a}} \mathrm{P}<0.05$. MAGE, melanoma-associated antigen-A; UICC, Union for International Cancer Control.

MAGE-A expression is significantly higher at the invasive front in UICC stage IV cancers. As shown in Table IV, the expression of all MAGE-A subgroups at the invasive front was higher in stage IV cancers than in stage I cancers. This finding was significant for MAGE-A1 ( $\mathrm{P}=0.0400),-\mathrm{A} 6(\mathrm{P}=0.0331)$, -A8 ( $\mathrm{P}=0.0434),-\mathrm{A} 9(\mathrm{P}=0.0060)$ and-A11 $(\mathrm{P}=0.0249)$.

Ratios of expression in the tumor center and invasive front are different in UICC stage I and stage IV cancers. To clarify possible changes in the MAGE-A expression pattern from limited to advanced disease, the ratio of MAGE-A expression at the tumor center compared with that at the invasive front ( $\mathrm{C} / \mathrm{F}$ ratio) was examined. As shown in Fig. 3, in stage I cancers, this ratio was always $>1.0$. The highest $\mathrm{C} / \mathrm{F}$ ratio in the stage I cancers was observed for MAGE-A11 (4.59). The lowest $\mathrm{C} / \mathrm{F}$ ratio was observed for MAGE-A3 (1.05). By contrast, in 9 out of the $11(81.8 \%)$ MAGE-A subgroups, the 

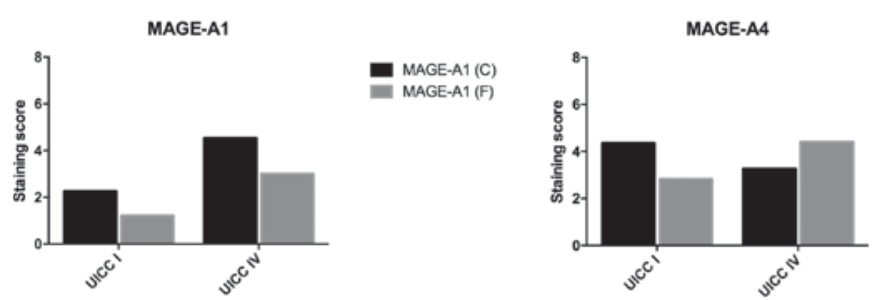

MAGE-A4 (C)
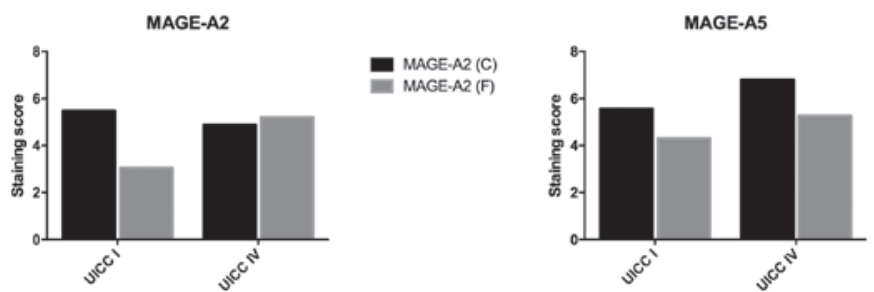

- MAGE-A5 (C)
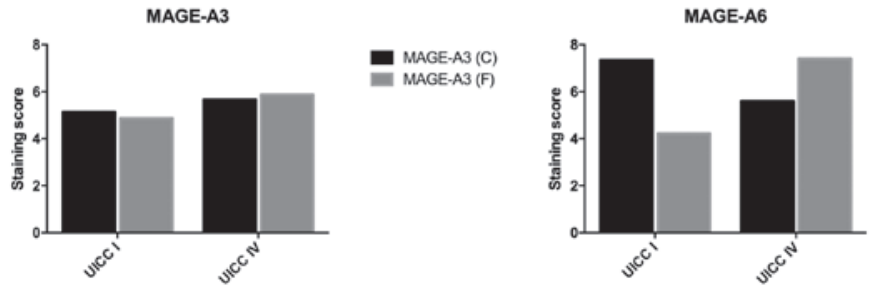

MAGE-A6 (C)

Figure 1. Different expression levels of MAGE-A1 to A6 at the tumor center and invasive front. In the tumor center, no significant differences could be observed between stage I and stage IV cancers. By contrast, MAGE-A1 $(\mathrm{P}=0.0400)$ and MAGE-A6 $(\mathrm{P}=0.0331)$ were expressed at significantly higher levels at the invasive front of stage IV cancers compared with stage I cancers. C, tumor center; F, invasive front; MAGE, melanoma-associated antigen-A; UICC, Union for International Cancer Control.
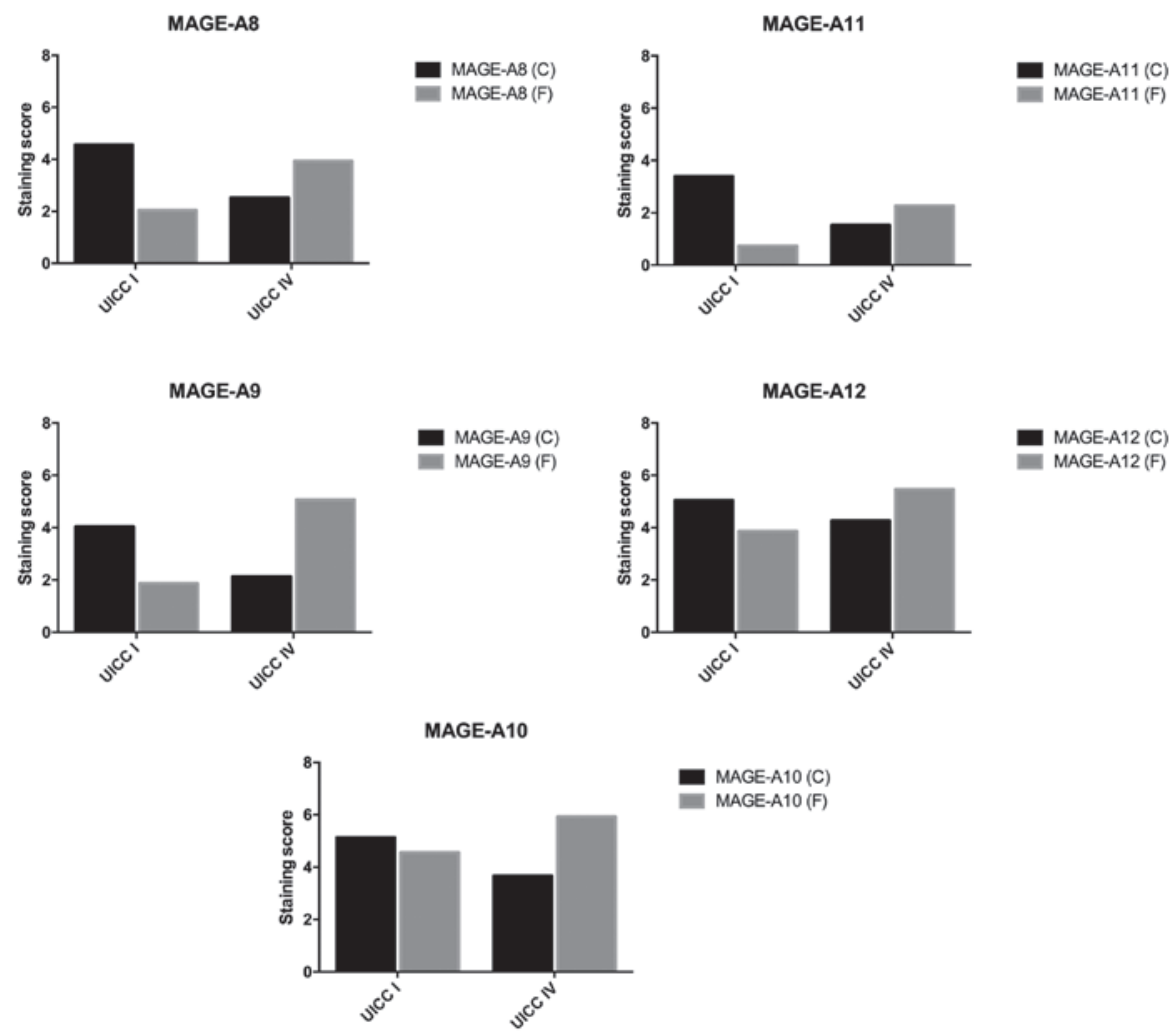

Figure 2. Different expression levels of MAGE-A8 to A12 at the tumor center and invasive front. No significant differences between stage I and stage IV cancers could be observed at the tumor center. By contrast, MAGE-A8 $(\mathrm{P}=0.0434),-\mathrm{A} 9(\mathrm{P}=0.0060)$ and $-\mathrm{A} 11(\mathrm{P}=0.0249)$ were all expressed at significantly higher levels at the invasive front of stage IV cancers compared with stage I cancers. C, tumor center; F, invasive front; MAGE, melanoma-associated antigen-A; UICC, Union for International Cancer Control. 


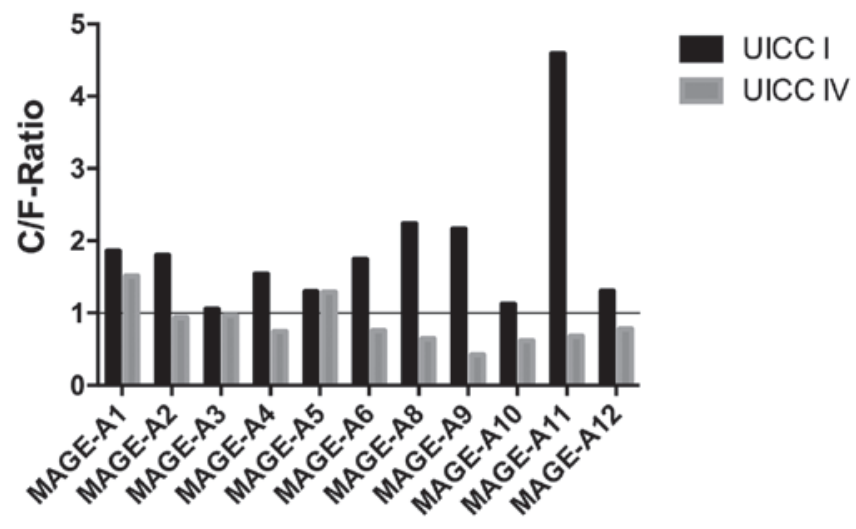

Figure 3. Ratio of the tumor center and invasive front ( $\mathrm{C} / \mathrm{F}$ ratio) expression of all MAGE-A subgroups in arbitrary units. In stage I cancers, the ratio was always $>1.0$. The highest $\mathrm{C} / \mathrm{F}$ ratio in stage $\mathrm{I}$ cancers was observed for MAGE-A11 (4.59). The lowest C/F ratio was observed for MAGE-A3 (1.05) By contrast, for 9/11 MAGE-A subgroups, the ratio was $<1.0$ in the UICC stage IV cancers. The highest $\mathrm{C} / \mathrm{F}$ ratio was observed for MAGE-A1 (1.51) and the lowest was observed for MAGE-A9 (0.42). The largest change in the $\mathrm{C} / \mathrm{F}$ ratio from stage I to stage IV was observed in MAGE-A11 (4.59 vs. 0.68)

ratio was $<1.0$ in the advanced cancers (UICC stage IV). The highest $\mathrm{C} / \mathrm{F}$ ratio was observed for MAGE-A1 (1.51), while the lowest was observed for MAGE-A9 (0.42). Thus, the largest change in the $\mathrm{C} / \mathrm{F}$ ratio from stage I to stage IV was observed in MAGE-A11 (4.59 vs. 0.68). The smallest change was observed in MAGE-A5 (1.2929 vs. 1.2911).

\section{Discussion}

To the best of our knowledge, this is the first study to investigate the changes in MAGE-A expression in the tumor front and the tumor center in the early and later stages of cancer. Considering the large number of studies that have investigated markers of malignancy in head and neck cancer (17-20), the 38 samples examined in the present study are adequate for a first overview, although large-scale studies will be required to confirm the findings. All investigated MAGE-A subgroups were detected in the stage I and IV cancers, although not every patient showed expression of all subgroups. At the tumor center of stage I cancers, MAGE-A6 expression yielded the highest staining. In an in vitro analysis, Müller-Richter et al also showed high levels of MAGE-A6 in comparison to other subgroups in a panel of established head and neck cancer cell lines (21). By contrast, the UICC stage IV cancers in the present study showed the highest expression of MAGE-A5 at the tumor center. This is important as we recently showed a correlation between a lower response to epidermal growth factor receptor (EGFR) antibodies (panitumumab) and the high expression of MAGE-A5 in several head and neck cancer cell lines (10). This is particularly significant as patients with advanced head and neck tumors are increasingly being treated with targeted therapies, including EGFR antibodies, indicating the clinical relevance of the finding. Investigating the MAGE-A5 status prior to the administration of anti-EGFR therapy could aid in supporting clinical decisions.

At the invasive front of limited head and neck cancers (UICC stage I), the highest staining rate was found for MAGE-A3 in the present study. Notably, MAGE-A3 has been widely described by previous studies and serves as a target for vaccination in the treatment of head and neck tumors $(22,23)$. These studies have shown encouraging results and shed light on novel treatment options for patients suffering from advanced tumors of the head and neck. In contrast to these previous studies, the present study detected MAGE-A6 as the most highly expressed at the invasive front of UICC stage IV cancers.

As the different functions and crosslinks among the 11 MAGE-A subgroups are not yet completely understood, the use of the cumulative MAGE-A expression as an element for distinguishing between limited and advanced stages of disease was considered in the present study. The cumulative expression was indeed higher in the UICC stage IV group (98.67 vs. 85.87), but this finding was not significant. By comparing the subgroup expression in the tumor center, higher staining was detected for MAGE-A1, -A3 and -A5 in the UICC stage IV cancers. For the other subgroups, the staining rates were higher in UICC stage I cancers. However, the difference was not significant for any of the subgroups tested. In clear contrast to that finding, higher staining was observed for all MAGE-A proteins in the invasive front for the UICC stage IV group. These findings were significant for MAGE-A1 ( $\mathrm{P}=0.0400),-\mathrm{A} 6(\mathrm{P}=0.0331),-\mathrm{A} 8(\mathrm{P}=0.0434),-\mathrm{A} 9(\mathrm{P}=0.0060)$ and $-\mathrm{A} 11(\mathrm{P}=0.0249)$.

A marked change was clearly present in the expression patterns in the tumor front and center during the progression of the studied malignancies. MAGE-A9 and-A11 are worthy of note in this context. Recently, Han et al provided evidence of a poor prognosis in patients with laryngeal squamous cell carcinomas expressing high levels of MAGE-A9 (24). In addition, we have previously provided strong evidence that MAGE-A11 is correlated with the reduced effects of numerous agents commonly used for the treatment of head and neck cancer, such as cisplatin, 5-fluorouracil, docetaxel, paclitaxel, cetuximab and panitumumab $(9,10)$. Furthermore, this finding has also been confirmed for erlotinib and gefitinib (25). Notably, comparable to our previous results in cell culture, the MAGE-A11 expression in the TMAs was only modest (25). However, its expression can likely be used to separate patients with a higher risk from those with a lower risk. As outlined for breast and prostate cancer $(11,26)$, MAGE-A11 may play a role in the growth and progression of head and neck cancer.

By investigating the tumor center and invasive front separately in limited and advanced stages of the disease, the present study was able to obtain the first insights into the changes of MAGE-A expression that occur during cancer progression. To the best of our knowledge, the present study is the first to describe a significant change in the MAGE-A pattern in head and neck cancer from UICC stage I to IV. By calculating the $\mathrm{C} / \mathrm{F}$ ratio for each MAGE-A subgroup, intense changes were identified from UICC stage I to IV. While the $\mathrm{C} / \mathrm{F}$ ratios in limited disease were always $>1.0$, indicating a stronger MAGE-A expression in the tumor center, this ratio changed in 9 out of 11 MAGE-A subgroups in samples of advanced disease. With the exception of MAGE-A1 and -A5, all other subgroups were more highly expressed at the invasive front of advanced-stage tumors. As an advanced stage based on the UICC criteria always represents distinct local invasion or regional/distant metastatic spread, the relocation of MAGE-A 
proteins from the tumor center to the invasive front could be considered to contribute to malignancy. Notably, this effect was the strongest for MAGE-A11, the subgroup previously identified as a predictor of decreased treatment efficacy in head and neck cancer cell lines. In summary, the present findings warrant further investigation into the MAGE-A proteins, particularly the role of MAGE-A11, in head and neck cancer.

\section{Acknowledgements}

Language editing support was provided by American Journal Experts.

\section{References}

1. Kamangar F, Dores GM and Anderson WF: Patterns of cancer incidence, mortality, and prevalence across five continents: Defining priorities to reduce cancer disparities in different geographic regions of the world. J Clin Oncol 24: 2137-2150, 2006

2. Torre LA, Bray F, Siegel RL, Ferlay J, Lortet-Tieulent J and Jemal A: Global cancer statistics, 2012. CA Cancer J Clin 65: 87-108, 2015.

3. Hamoir M, Schmitz S and Gregoire V: The role of neck dissection in squamous cell carcinoma of the head and neck. Curr Treat Options Oncol 15: 611-624, 2014.

4. van der Bruggen $\mathrm{P}$, Traversari $\mathrm{C}$, Chomez $\mathrm{P}$, Lurquin $\mathrm{C}$, De Plaen E, Van den Eynde B, Knuth A and Boon T: A gene encoding an antigen recognized by cytolytic $\mathrm{T}$ lymphocytes on a human melanoma. Science 254: 1643-1647, 1991.

5. Abd-Elsalam EA and Ismaeil NA: Melanoma-associated antigen genes: A new trend to predict the prognosis of breast cancer patients. Med Oncol 31: 285, 2014.

6. Laban S, Atanackovic D, Luetkens T, Knecht R, Busch CJ, Freytag M, Spagnoli G, Ritter G, Hoffmann TK, Knuth A, et al: Simultaneous cytoplasmic and nuclear protein expression of melanoma antigen-A family and NY-ESO-1 cancer-testis antigens represents an independent marker for poor survival in head and neck cancer. Int J Cancer 135: 1142-1152, 2014.

7. Li G, Song P and Zhang B: Expression and significance of MAGE genes in human lung cancer. Zhongguo Fei Ai Za Zhi 16: 308-313, 2013.

8. Monte M, Simonatto M, Peche LY, Bublik DR, Gobessi S, Pierotti MA, Rodolfo M and Schneider C: MAGE-A tumor antigens target p53 transactivation function through histone deacetylase recruitment and confer resistance to chemotherapeutic agents. Proc Natl Acad Sci USA 103: 11160-11165, 2006.

9. Hartmann S, Kriegebaum U, Küchler N, Brands RC, Linz C Kübler AC and Müller-Richter UD: Correlation of MAGE-A tumor antigens and the efficacy of various chemotherapeutic agents in head and neck carcinoma cells. Clin Oral Investig 18 $189-197,2014$

10. Hartmann S, Kriegebaum U, Küchler N, Lessner G, Brands RC, Linz C, Schneider T, Kübler AC and Müller-Richter UD: Efficacy of cetuximab and panitumumab in oral squamous cell carcinoma cell lines: Prognostic value of MAGE-A subgroups for treatment success. J Craniomaxillofac Surg 41: 623-629, 2013.

11. Xia LP, Xu M, Chen Y and Shao WW: Expression of MAGE-A11 in breast cancer tissues and its effects on the proliferation of breast cancer cells. Mol Med Rep 7: 254-258, 2013.
12. Minges JT, Su S, Grossman G, Blackwelder AJ, Pop EA, Mohler JL and Wilson EM: Melanoma antigen-A11 (MAGE-A11) enhances transcriptional activity by linking androgen receptor dimers. J Biol Chem 288: 1939-1952, 2013.

13. Ries J, Agaimy A, Vairaktaris E, Gorecki P, Neukam FW, Strassburg LH and Nkenke E: Detection of MAGE-A expression predicts malignant transformation of oral leukoplakia. Cancer Invest 30: 495-502, 2012.

14. Krauss E, Rauthe S, Gattenlöhner S, Reuther T, Kochel M, Kriegebaum U, Kübler AC and Müller-Richter UD: MAGE-A antigens in lesions of the oral mucosa. Clin Oral Investig 15: 315-320, 2011.

15. Greene FL, Page DL, Fleming ID, Fritz A, Balch CM, Haller DG and Morrow M: AJCC cancer staging manual. 6th edition. Springer, New York, 2002.

16. Remmele W and Stegner HE: Recommendation for uniform definition of an immunoreactive score (IRS) for immunohistochemical estrogen receptor detection (ER-ICA) in breast cancer tissue. Pathologe 8: 138-140, 1987 (In German).

17. Hildebrand LC, Carvalho AL, Lauxen IS, Nör JE, Cerski CT and Sant'Ana Filho M: Spatial distribution of cancer stem cells in head and neck squamous cell carcinomas. J Oral Pathol Med 43: 499-506, 2014.

18. Lindberg P, Larsson A and Nielsen BS: Expression of plasminogen activator inhibitor-1, urokinase receptor and laminin gamma-2 chain is an early coordinated event in incipient oral squamous cell carcinoma. Int J Cancer 118: 2948-2956, 2006.

19. Ries J, Schultze-Mosgau S, Neukam F, Diebel E and Wiltfang J: Investigation of the expression of melanoma antigen-encoding genes (MAGE-A1 to-A6) in oral squamous cell carcinomas to determine potential targets for gene-based cancer immunotherapy. Int J Oncol 26: 817-824, 2005.

20. van den Brand M, Takes RP, Blokpoel-deRuyter M, Slootweg PJ and van Kempen LC: Activated leukocyte cell adhesion molecule expression predicts lymph node metastasis in oral squamous cell carcinoma. Oral Oncol 46: 393-398, 2010.

21. Muller-Richter UD, Dowejko A, Reuther T, Kleinheinz J, Reichert TE and Driemel O: Analysis of expression profiles of MAGE-A antigens in oral squamous cell carcinoma cell lines. Head Face Med 5: 10, 2009.

22. Voskens CJ, Sewell D, Hertzano R, DeSanto J, Rollins S, Lee M, Taylor R, Wolf J, Suntharalingam M, Gastman B, et al: Induction of MAGE-A3 and HPV-16 immunity by Trojan vaccines in patients with head and neck carcinoma. Head Neck 34: 1734-1746, 2012.

23. Zandberg DP, Rollins S, Goloubeva O, Morales RE, Tan M, Taylor R, Wolf JS, Schumaker LM, Cullen KJ,Zimrin A, et al: A phase I dose escalation trial of MAGE-A3-and HPV16-specific peptide immunomodulatory vaccines in patients with recurrent/metastatic (RM) squamous cell carcinoma of the head and neck (SCCHN). Cancer Immunol Immunother 64: 367-379, 2015

24. Han L, Jiang B, Wu H, Zhang S and Lu X: Expression and prognostic value of MAGE-A9 in laryngeal squamous cell carcinoma. Int J Clin Exp Pathol 7: 6734-6742, 2014.

25. Hartmann S, Brands RC, Küchler N, Fuchs A, Linz C, Kübler AC and Müller-Richter UD: Melanoma-associated antigen expression and the efficacy of tyrosine kinase inhibitors in head and neck cancer. Oncol Lett 10: 1211-1217, 2015.

26. Wilson EM: Androgen receptor molecular biology and potential targets in prostate cancer. Ther Adv Urol 2: 105-117, 2010 . 